\title{
Angiographic Pattern of Coronary Artery Stenosis in Type 2 Diabetic Patients with Acute Coronary Syndrome
}

\begin{abstract}
Abu Tarek lqbal ${ }^{1}$
Abdul Jalil ${ }^{2}$

$M$ Jalal Uddin ${ }^{3 *}$
\end{abstract}

'Department of Medicine

Chattogram Maa-O-Shishu Hospital Medical College Chattogram, Bangladesh.

${ }^{2}$ Department of Cardiology Chattogram General Hospital

Chattogram, Bangladesh.

${ }^{3}$ Department of Community Medicine Chattogram Maa-O-Shishu Hospital Medical College Chattogram, Bangladesh.
*Correspondence to:

Professor (Dr) M. Jalal Uddin

Head, Department of Community Medicine

Chattogram Maa-O-Shishu Hospital Medical College

Chattogram, Bangladesh.

Mobile : +8801819909464

Email :drialal65@gmail.com

\section{Date of Submission : 15.02 .2020 \\ Date of Acceptance : $\quad 25.07 .2020$}

www.banglajol.info/index.php/CMOSHMCJ

\begin{abstract}
Background: Many studies were conducted in home and abroad on the subject but there is none in Chattogram region of Bangladesh. To unveil the existing situation we have conducted the study which will help better case management.

Materials and methods: Properly diagnosed 54 consecutive type 2 diabetic patients with Acute Coronary Syndrome were selected for study. The study was conducted in 2016 (One year) at a private cardiac center of Chattogram city. After adequate preparation angiography was performed with standard Judkin's technique. Data were manually managed and statistically analyzed. Results were compared with other similar studies. Informed consent was taken from the patients and the hospital authority before conducting the study. A competent institutional review board approved the protocol.
\end{abstract}

Results: Sex of the patients were male $85 \%$ and female $15 \%$. Age range was $30-70$ years. Peak age group was 50-60 years. It was $41 \%$. Mean age was $56 \pm 6.8$ years. Multiple Vessels Disease (MVD) was the highest 39\%. Commonly affected vessel was Left Anterior Descending artery (LAD) 83\%.

Conclusion: Diabetes mellitus is a notorious risk factor for coronary artery disease. It causes coronary artery lesions earlier. It significantly causes acute coronary syndrome. So all measures should be taken to prevent and control diabetes mellitus.

Key words: Coronary artery disease; Coronary angiogram; Diabetes mellitus.

\section{INTRODUCTION}

Type 2 diabetes mellitus is the number 2 risk factor for coronary artery stenosis ${ }^{1}$. It increases risk of coronary artery disease by $2-3$ times higher than non diabetic patients ${ }^{2}$. The prevalence of coronary artery disease is not only high but also more severe in diabetic patient than that of non diabetic patients ${ }^{3}$. Other factors for coronary artery disease are : Hypertension, smoking, Dyslipidemia and positive family histo$\mathrm{ry}^{1}$. Even after control of other risk factors type- 2 diabetes mellitus alone increases number of stenosis of coronary arteries ${ }^{4}$. The relationship between coronary artery disease and diabetes mellitus was established by Seegen $\mathrm{J}$ in $1870^{2}$.

Acute coronary syndrome encompasses both unstable angina and Myocardial Infarction (MI). It is characterized by new onset or rapidly worsening angina on minimal exertion or angina at rest in absence of myocardial damage. In contrast MI occurs when symptoms are manifested at rest and there is evidence of myocardial necrosis as demonstrated by an elevation of troponin or CK-MB isoenzyme ${ }^{5}$. Diagnosis of Acute Coronary Syndrome was done on the basis of history, sign symptoms, biomarkers ECG and echocardiography evaluation. 


\section{MATERIALS AND METHODS}

A total of 54 type -2 diabetic patients with acute coronary syndrome were selected on the basis of history, clinical features, biomarkers, ECG and echocardiography. The study was conducted in 2017 at a private cardiac center of Chattogram. Critical patients were excluded from the study. After adequate preparation angiography was performed with standard Judkin's technique. Data were manually managed and t test was used for analysis. Results were compared with previous similar studies. Informed consent was taken from patient and hospital authority. The protocol was approved by a competent Institutional review board.

\section{RESULTS}

Among the 54 patients male were $85 \%$ and female were $15 \%$. Age range of the respondents was 30-70 years. Peak age group was $50-60$ years. It was $41 \%$ in male and $50 \%$ in female. Average $45.5 \%$. Mean age was $56 \pm 6.8$ years. Multiple Vessel Disease (MVD) was the highest $39 \%$. Single vessel disease was $22 \%$ and double vessel disease was $20 \%$. Commonly affected vessel was Left Anterior Descending (LAD) artery. It was $83 \%$. Left Circumflex artery $\left(\mathrm{LC}_{\mathrm{X}}\right)$ stenosis was the second $66 \%$ and Right Coronary Artery (RCA) was the third position holder 58\%.

Table I : Age and sex of respondents.

\begin{tabular}{lcr} 
Age groups & Male respondents & Female respondents \\
$30-40$ Years & $07(15 \%)$ & $02(25 \%)$ \\
$40-50$ Years & $12(26 \%)$ & $01(12.5 \%)$ \\
$50-60$ Years & $19(41 \%)$ & $04(50 \%)$ \\
$60-70$ Years & $08(17 \%)$ & $01(12.5 \%)$ \\
Total & $46(85 \%)$ & $08(15 \%)$ \\
\hline
\end{tabular}

Source: CMPH records 2016.

Table II : Number of stenosed vessels.

\begin{tabular}{lc} 
Number of vessels stenosed & Frequency (\%) \\
No stenosis & $10(19 \%)$ \\
Single vessel disease & $12(22 \%)$ \\
Double vessel disease & $11(20 \%)$ \\
Multiple vessel disease & $21(39 \%)$ \\
Total & $54(100 \%)$ \\
\hline
\end{tabular}

Table III : Ranking of stenosed vessels among male patients.

\begin{tabular}{llc} 
Ranking & Name of vessel & Frequency (\%) \\
01 & LAD & $30(83 \%)$ \\
02 & LCx & $24(66 \%)$ \\
03 & RCA & $21(58 \%)$ \\
04 & LMCA & $01(03 \%)$ \\
\hline
\end{tabular}

Source: CMPH records 2016

\section{DISCUSSION}

Average age of respondents of present study was $56 \pm 6.8$ years. Similar result was found in the study conducted by Barzani ${ }^{2}$. It was $59.4 \pm 9.2$ years. There was difference regarding sex in between above studies (Male $85 \%$ VS $49.3 \%$ and female 15\% VS $49.3 \%)$. The difference is significant $(\mathrm{p}=0.02)$. In both the studies triple vessel disease ranked the highest position (39\% VS $60 \%)$. The difference was significant $(\mathrm{p}=.02)$. According to Verghes $\mathrm{K}$ et al it was $27 \%{ }^{6}$. About ranking of stenosed vessel left anterior descending artery ranked the first position in both the studies $(83 \%$ VS $89 \%)$. However the differences was not significant $(\mathrm{p}=0.1)$.

\section{CONCLUSION}

Diabetes mellitus is the number 2 risk factor for development of coronary artery disease. It causes stenosis earlier and number of vessel involvement and severity of stenosis both are enhanced by diabetes mellitus type 2 . So all out measures should be taken to prevent and control diabetes mellitus.

\section{DISCLOSURE}

All the authors declared no competing interest.

Source: CMPH records 2016.

\section{REFERENCES}

1. Iqbal A.T., Uddin M.J., Mamun S.M.H., Biswas R.S.R. Top 3 risk factors of coronary artery disease. Chattogram Maa-O-Shishu Hospital Medical College Journal. 2017; 16(1): 26-28.

2. Albarzani M.A. Angiographic profile in diabetic and non diabetic patients with coronary artery disease in the cardiac specialty Hospital.Zanco J. Med. Sci. 2017; 21(2): 1701-1707.

3. Srinivasan M.P et al. Severity of coronary artery disease in type 2 diabetes mellitus: Does timing matter. Available at: www.ncbi.nlm.nih.gov/pmc/articles/PMC 4867948/

4. Newby D.E., Grubb N.R., Brodbuxy A. Cardiovascular disease.In : Walker B.R., Colledge N.R., Ralston S.H., Perman I.D., editors. Davidsons principle and practice of medicine. New Delhi: Churchill Livingstone. 2010;589-590.

5. Arad Y., Newslein D., Cadet F., Roth M., Guerci A.D. Association of multiple risk factors and insulin resistance with increased prevalence of asymptomatic coronary artery disease by an electron beam computed Tomography. ArteriosclerThromb vase Biol. 2001; 21:2051-2058.

6. Varghese K., Cherian G., Abraham M.T., Hayat N.J. Johny K.V. Coronary artery disease among diabetic and non diabetic patients with end stage renal diseases. Available at: www.ncbi.nlm.nih.gov/pubmed/11725913. 